\title{
Combination therapy with pulsed methylprednisolone in rheumatoid arthritis
}

\author{
V NEUMANN ${ }^{1}$ R HOPKINS,${ }^{1}$ J DIXON ${ }^{2}$ A WATKINS ${ }^{3}$ H BIRD ${ }^{2}$ AND \\ V WRIGHT
}

From the ' Rheumatism Research Unit, University of Leeds; the ${ }^{2}$ Clinical Pharmacology Unit, Royal Bath Hospital, Harrogate; and the ${ }^{3}$ Department of Statistics, University of Leeds

SUMmaRY Pulsed methylprednisolone (PMP) has been shown to produce clinical improvement and reduction in the ESR and acute phase protein concentrations in patients with active rheumatoid arthritis and has been advocated for use either as an alternative to slow-acting antirheumatoid drugs (SAARDs) or in conjunction with SAARDs to accelerate the response to treatment. To test these potential roles for PMP 45 patients with active RA were randomly allocated to treatment with PMP alone, PMP+sulphasalazine (SAS - at a maintenance dose of $2.0 \mathrm{~g} /$ day), or PMP + D-penicillamine (DPA - at a maintenance dose of $500 \mathrm{mg} / \mathrm{day}$ ). In each case three $1 \mathrm{~g}$ intravenous infusions were given on alternate days during the first week of the trial. Patients were monitored for 24 weeks by standard clinical and laboratory measurements. All three treatment groups showed significant clinical and laboratory improvements at two weeks. With PMP+DPA and PMP+SAS these improvements were sustained and were not significantly different in these two treatment groups. However, in the 'PMP only' group ESR and CRP rose to pretreatment values by eight weeks. Twelve patients withdrew from the study owing to a relapse of the RA. No serious adverse effects were seen in the 'PMP only' group. Both combination regimens were well tolerated; adverse effects seen were attributable to either DPA or SAS. We conclude that PMP alone is insufficient for treatment of RA but can be used successfully in combination with either DPA or SAS. A comparison between these results and results obtained from two previous groups of 15 patients treated with DPA alone and SAS alone (using the same study design) shows that PMP accelerated the response to therapy by at least six weeks.

Intravenous 'pulsed' methylprednisolone therapy (PMP) has been reported to produce clinical improvement in active rheumatoid arthritis (RA) and reduction of the erythrocyte sedimentation rate (ESR) and C-reactive protein concentrations (CRP). ${ }^{1}{ }^{2}$ In the first of these two studies ${ }^{1}$ patients were given single $1 \mathrm{~g}$ pulses. This produced clinical improvement lasting six weeks, though ESR and CRP were only improved significantly for two weeks. In the second study ${ }^{2}$ three $1 \mathrm{~g}$ pulses were given on alternate days over a five-day period. This produced clinical improvement lasting a mean of 10 weeks; biochemical improvement was maintained for seven weeks. Both groups of workers suggested two potentially useful roles for PMP. Repeated

\footnotetext{
Accepted for publication 30 April 1985.

Correspondence to $\mathrm{Dr} \mathrm{V}$ Neumann, Rheumatology and Rehabilitation Research Unit, School of Medicine, 36 Clarendon Road, Leeds LS2 9PJ.
}

pulses were suggested for patients with persistently active disease who either fail to respond to conventional long-term therapy such as gold or penicillamine or have been unable to tolerate these drugs. PMP was also advocated to 'bridge the gap' between the introduction of slow-acting antirheumatoid drugs and the delayed response to such drugs.

The present study was designed to examine the latter role for PMP in groups of patients with active RA. Because of the paucity of biochemical data about the use of PMP in RA, we also re-examined a group of patients given PMP alone.

\section{Patients and methods}

Forty-five patients with classical or definite RA were recruited from the outpatient clinic. All had 'active' disease as defined by the presence of three or more of the following characteristics: (a) tender- 
ness of more than six joints; $(b)$ swelling of more than three joints; $(c)$ early morning stiffness lasting more than 15 minutes; $(d)$ Ritchie articular index greater than 20; (e) ESR greater than $28 \mathrm{~mm} / 1 \mathrm{st} \mathrm{h}$.

Patients already taking steroids (other than local injections), patients who had received either sulphasalazine (SAS) or D-penicillamine (DPA) at any time in the past or who had received any other longterm agent within the preceding three months were excluded from the study, as were patients with severe dyspepsia, intercurrent infection, or any other disorder likely to be adversely affected by steroids. All had had RA for at least six months.

All 45 patients were assessed by the following clinical and laboratory tests: grip strength, change score, pain score (1-5), early morning stiffness, Ritchie articular index, joint size, functional grade (1-5), full blood count, viscosity, $\gamma$-glutamyltransferase, sulphydryl, histidine, CRP, fibrinogen, protein+albumin, IgM, urinaly̆sis.

After a two-week observation period on antiinflammatory drugs alone (usually diclofenac or naproxen) all the patients were reassessed and then placed in one of three treatment groups by a random number series. The first of these groups (PMP) alone) was admitted to hospital and given three $1 \mathrm{~g}$ methylprednisolone infusions on alternate days over a five-day period. Each infusion was given over 45 minutes. The patients were then discharged from hospital and monitored in the outpatient clinic. They were offered further courses of PMP if they relapsed and once again fulfilled admission requirements for the study, provided that at least four weeks had elapsed since their previous course of

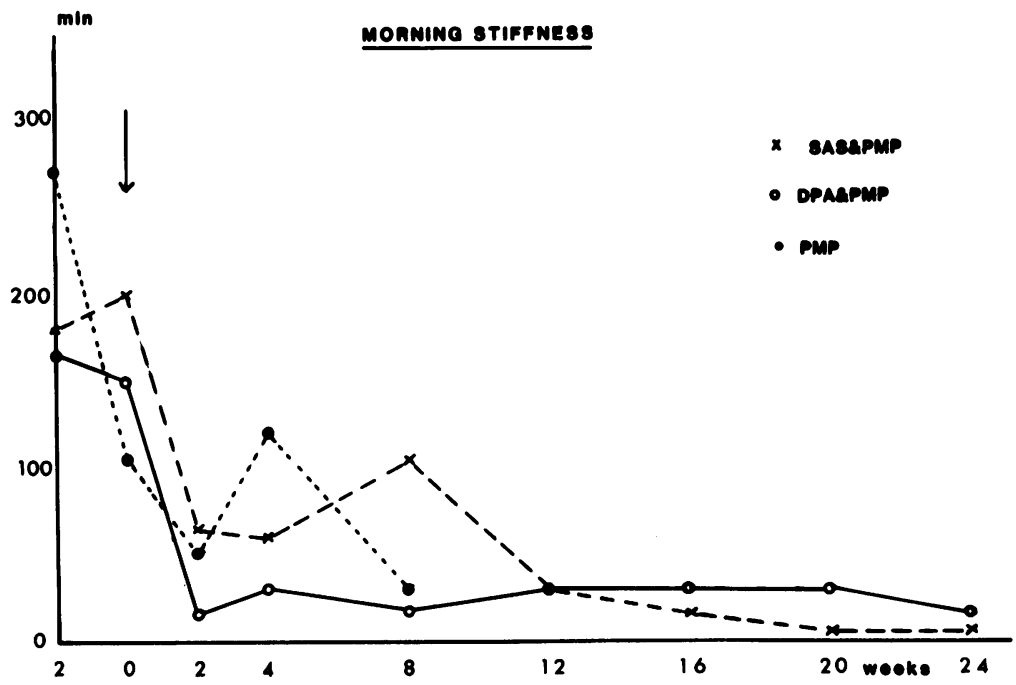

PMP. Groups 2 and 3 (PMP+DPA and PMP+SA were also initially admitted and given three 1 og pulses of methylprednisolone as described above On admission to hospital the PMP+DPA treatme group started DPA also at $125 \mathrm{mg} /$ day building up a maintenance dose of $500 \mathrm{mg} /$ day over six week홍 The PMP+SAS group instead started enteric-coate SAS $0.5 \mathrm{~g} /$ day building up to a maintenance dose $2 \mathrm{~g} /$ day over three weeks.

Patients were assessed at two weeks, then at fous weekly intervals over a 24 -week period. Treatmen? groups were compared by Kruskal-Wallis ant Mann-Whitney U tests. Response to treatment individual treatment groups was assessed by th: paired Mann-Whitney $U$ test to compare values as week 0 with values at subsequent visits for each variable.

\section{Results}

Patient characteristics were well matched in the three treatment groups as shown in Table 1.

Table 2 shows pretreatment disease activity in the

Table 1 Patients' characteristics

\begin{tabular}{lllll}
\hline & $P M P$ & $P M P+D P A$ & $P M P$ \\
\hline Male/female & $5 / 10$ & $5 / 10$ & $6 / 9$ \\
Age (years) & 56 & 48 & 53 & 9 \\
$\begin{array}{l}\text { Duration of disease } \\
\quad \text { (years) }\end{array}$ & 8 & 4 & 4 & 9 \\
Patients with nodules & 6 & 3 & & \\
RA latex positive & 10 & 10 & & \\
\hline
\end{tabular}

Fig. 1 Change in morning stiffnes with time. 
Table 2 Pretreatment disease activity*

\begin{tabular}{lrcc}
\hline & $P M P$ & $P M P+D P A$ & $P M P+S A S$ \\
\hline Morning stiffncss (min) & 108 & 150 & 200 \\
Ritchic articular index & 22 & 21 & 20 \\
ESR (mm/1st h) & 47 & 44 & 55 \\
CRP (mg/dl) ${ }^{-}$ & $3 \cdot 6$ & $3 \cdot 2$ & $2 \cdot 6$ \\
\hline
\end{tabular}

${ }^{*}$ Medians are shown.

†SI conversion: $\mathrm{mg} / \mathrm{dl} \times 10=\mathrm{mg} / \mathrm{l}$. three groups. The differences between the three groups before the start of therapy were not significant (Kruskal-Wallis, $\mathrm{p}>0.05$ ).

Our most striking finding was that 'PMP alone' proved an unacceptable treatment for the majority of our patients. Only two patients completed the 24week study. One patient was withdrawn from the study because of a myocardial infarct, thought not to be connected with therapy. The other 12 relapsed within eight weeks, and of these only four were prepared to accept repeated PMP courses. Three of

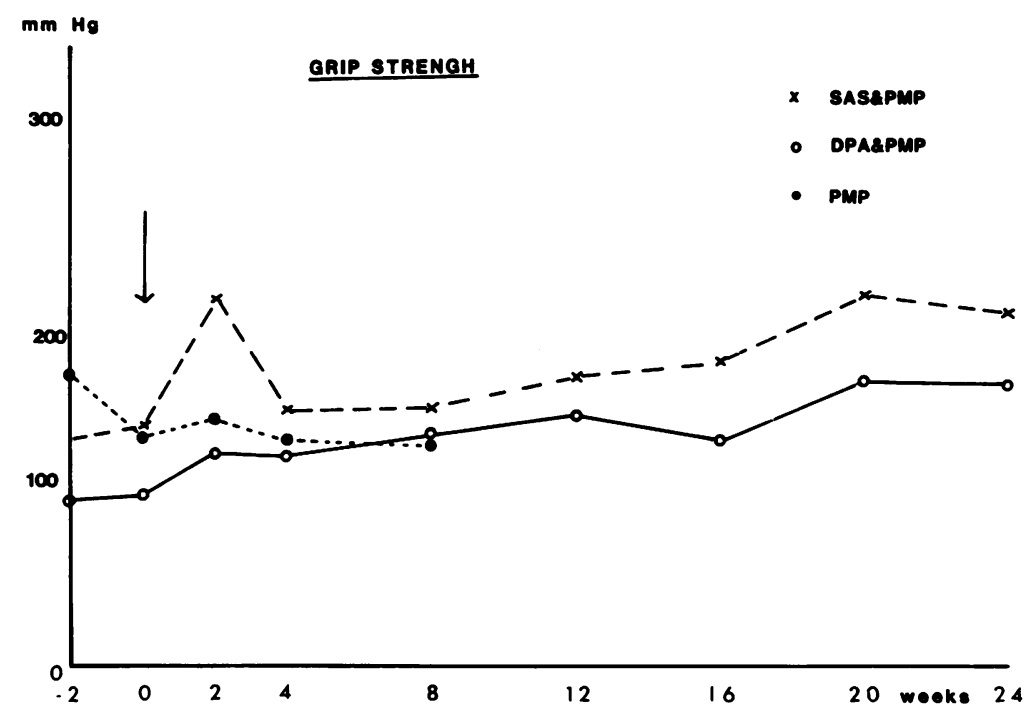

Fig. 2 Change in grip strength with time.

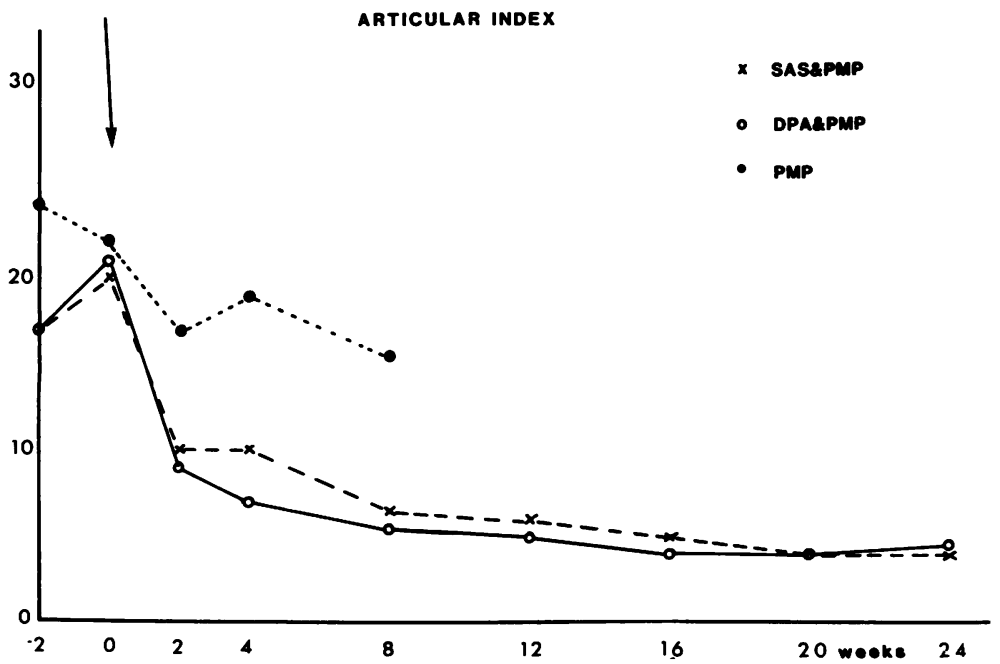

Fig. 3 Change in articular index with time. 
750 Neumann, Hopkins, Dixon, Watkins, Bird, Wright

these patients relapsed again and refused further PMP.

A combination of PMP+DPA was better tolerated. Four patients in this group failed to complete the study: three due to thrombocytopenia and one due to proteinurea, both of which could be attributed to DPA therapy. Seven patients dropped out of the PMP+SAS group: two because treatment was ineffective, three with severe dyspepsia, one with a rash, and one due to a family bereavement.
All three treatnıent groups showed substantia and significant clinical and laboratory improvemern when they were first assessed two weeks after the start of the treatment. The changes in morning stiffness, grip strength, articular index, ESR, an CRP are shown in Figs 1-5. As shown in thes? figures the initial significant improvements in the PMP+DPA and PMP+SAS were maintained and continued to improve over the 24 weeks of thes study.

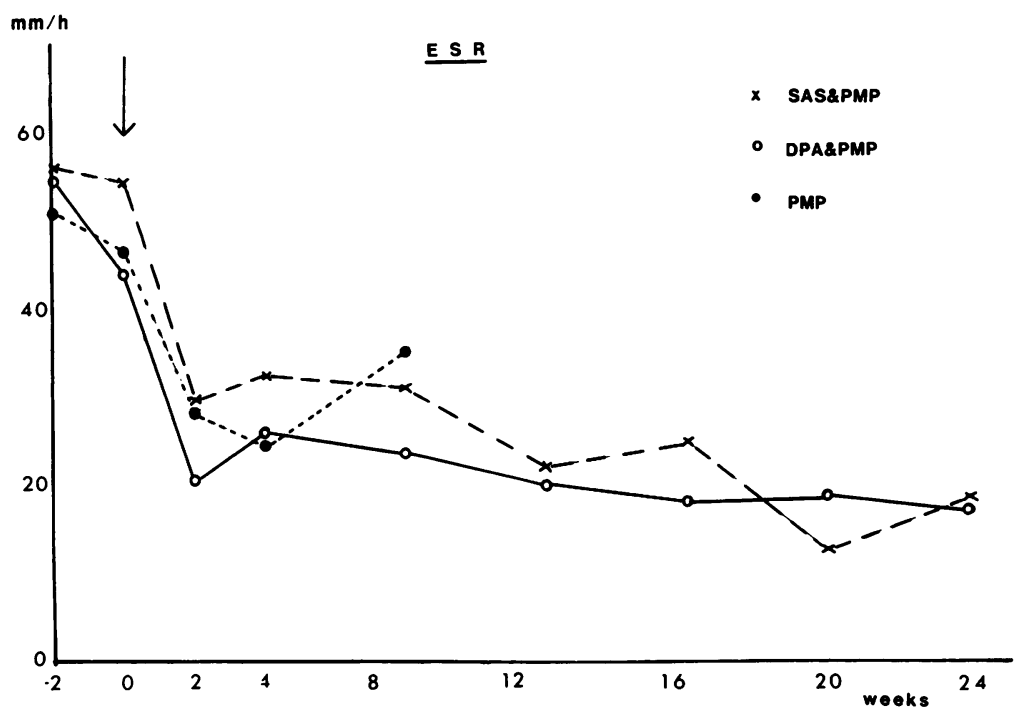

Fig. 4 Change in ESR with time

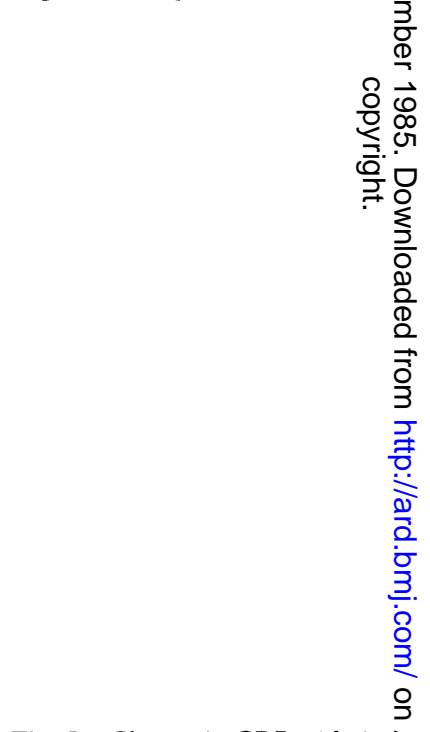

Fig. 5 Change in CRP with time (SI conversion: $m g / d l \times 10=m g / l$.

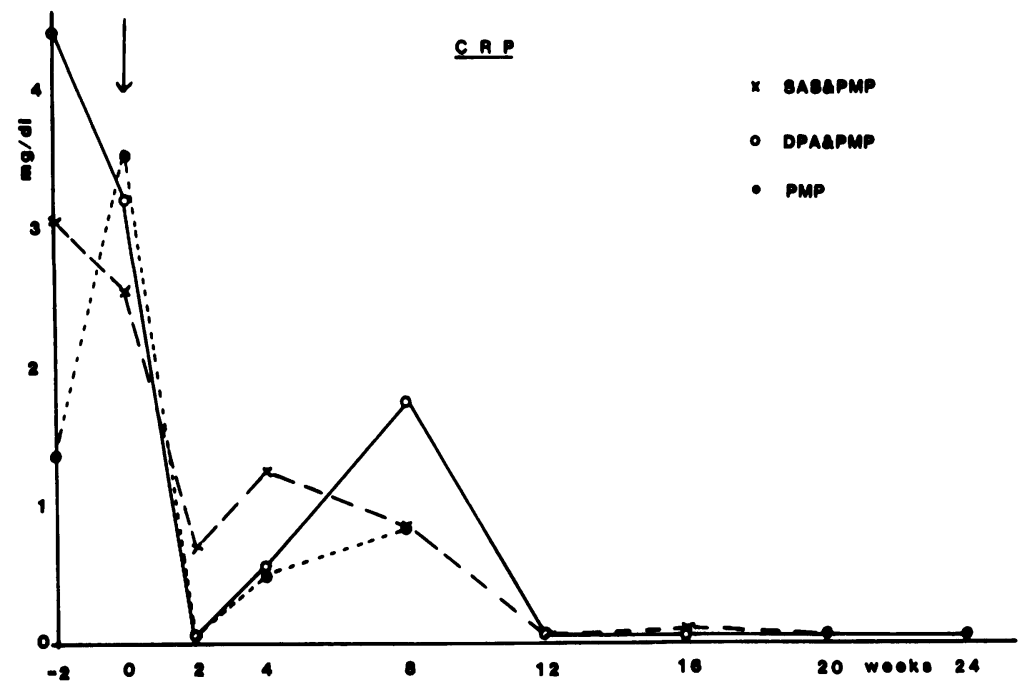




\section{Discussion}

Although our study confirmed previous observations that PMP given as three $1 \mathrm{~g}$ pulses can improve active RA both clinically and biochemically, it showed that this improvement was too short lived to be acceptable to the majority of rheumatoid patients. It should only be considered as an alternative treatment in patients with RA who have already failed to tolerate or respond to alternative, longterm agents. In contrast, PMP proved successful in combination with either SAS or DPA. We have compared our results with those obtained from two earlier studies of groups of 15 patients with active RA. In one of these DPA was given alone with the same dosage regimen as in the present study. In the other study SAS was given at a maintenance dose of 2-3 g/day. These two earlier studies used the same recruitment criteria and methods of assessment, and biochemical data were analysed in the same laboratories as in the present study. DPA alone produced little improvement until 16 weeks after the start of the study, and SAS when given alone showed substantial improvement only after eight weeks. Comparison suggests that the use of PMP in addition to these therapies thus accelerated the response to treatment by at least six weeks. At 24 weeks the overall response to either DPA or SAS was not altered by the addition of PMP.

Various serious side effects have been related to methylprednisolone pulse therapy, including sudden cardiac arrest ${ }^{3}$ and acute anaphylaxis. ${ }^{4}$ However, we observed no serious adverse effects due to PMP: those we saw could be attributed to either SAS or
DPA. The question remains, whether short courses of high-dose oral prednisolone would be as effective as PMP as well as being safer, cheaper, and more convenient to administer. The use of PMP in rheumatoid arthritis stems from its successful use in renal medicine for the treatment of allograft rejection episodes. In a recent study ${ }^{5}$ when high-dose methylprednisolone $\left(600 \mathrm{mg} / \mathrm{m}^{2}\right)$ was compared with low-dose oral prednisolone $(3 \mathrm{mg} / \mathrm{kg})$ for the treatment of such rejection episodes in children both therapies were equally effective and equally well tolerated.

In conclusion, our study has shown an effective use of PMP in combination with slow-acting antirheumatoid drugs in active RA. However, we have yet to show that this combination is superior to a combination of oral steroids and slow-acting antirheumatoid drugs and are now testing this alternative.

\section{References}

1 Williams I A. Baylis E M. Shipley M E. A double-blind placebo-controlled trial of methylprednisolone pulse therapy in active rhcumatoid discasc. Lancet 1982; ii: 237-40.

2 Forster P J G, Grindulis K, Neumann V, Hubball S, McConkey B. High-dose intravenous methylprednisolone in rheumatoid arthritis. Ann Rheum Dis 1982; 41: 444-6.

3 Barrett D F. Pulse methylprednisolone therapy. Lancet 1983; ii: $8(x)$.

4 Prysc-Phillips W E M. Chandra R K. Rosc B. Anaphylactoid reaction to methylprednisolone pulsed therapy for multiple sclcrosis. Neurlogy (NY) 1984: 34: 1119-21.

5 Orta-Sibu N. Chantler C. Bewick M, Aaycock G. Comparison of high-dose IV methylprednisolone with low-dosc oral prednisolone in acute renal allograft rejection in children. Br Med J 1982: 285: 258-60).

\section{Book review}

Aspects of Manipulative Therapy. 2nd edn. Eds. E F Glasgow, L W Twomey, E R Scull, A M Kleynhans, R M Idczak. Pp. 194. £14.95. Churchill Livingstone: Edinburgh. 1984.

This book will have a limited readership, but it contains several good chapters, particularly on radiology and computed tomographic scanning. Unfortunately the impression it gives that sacroiliitis is a common manifestation of rheumatoid arthritis spoils the overall validity. The mythology is perpetuated that radiology is essential before manipulative therapy; actually it may give a false sense of security and be used instead of full clinical assessment. I would consider the presence of inflammatory joint disease a contraindication to neck manipulation even with normal $x$-rays. In the chapter on neck and shoulder pain the section on pain from periarticular lesions of the shoulder is inaccurate and misleading. 